\title{
COMPOR: a Methodology, a Component Model, a Component based Framework and Tools to Build Multiagent Systems
}

\author{
Hyggo Oliveira de Almeida ${ }^{1 *}$, Angelo Perkusich ${ }^{1}$ \\ Evandro de Barros Costa ${ }^{2}$ and Rodrigo de Barros Paes ${ }^{3}$ \\ ${ }^{1}$ Electrical Engineering Departament \\ Federal University of Paraíba \\ Caixa Postal 10105, 58109-970, Campina Grande, PB, Brazil \\ \{hyggo,perkusic\}@ dee.ufcg.edu.br \\ ${ }^{2}$ Department of Information Technology \\ Federal University of Alagoas, Maceió, AL, Brazil \\ ebc@fapeal.br \\ ${ }^{3}$ Department of Informatics \\ Pontifical Catholic University, Rio de Janeiro, RJ, Brazil \\ rbp@les.puc-rio.br
}

\begin{abstract}
In this paper a set of guidelines, techniques and tools to develop software for multiagent systems (MAS), named COMPOR, are introduced. The main objective is to provide an effective approach to deal with the inherent dynamic and openness characteristics of such systems. COMPOR is based on a conceptual model for a society of agents named MATHEMA and on the component based software development. We present a methodology, a component model, a framework, and an environment to aid during the development process of MAS. We also discuss the application of COMPOR for an electronic commerce negotiation problem.
\end{abstract}

\section{Introduction}

Agent and multiagent technologies have been investigated in the last years as a promising approach to the modelling, analysis and design of complex software systems [28]. Even so, there is no consensus with respect to a clear definition of the term "agent". Based on the definitions given by Russel [45], Ferber [22] and Jennings [35], in the context of this paper, we define an agent as a problem solving entity, that tracks changes in the environment based on the information from sensors and performs autonomous and flexible actions by means of actuators. Also, an agent can interact with other agents in order to reach goals. Based on this definition it is possible to identify the following properties for an agent [46]: autonomy is the capability of an agent to operate without the intervention of humans and other agents, and to execute

\footnotetext{
${ }^{*} \mathrm{PhD}$ student at the Electrical Engineering Doctorate Program, COPELE/DEE/UFCG, sponsored by CNPq (Brazilian National Research Council).
} 
some kind control over its actions; rationality means that the behavior of an agent is guided by objectives; reactivity is the skill to detect and react to changes in the environment; pro-activity means that an agent may start to search its goals instead of only reacting to demands; social skill is the capability of an agent to interact with other agents based on a given communication language in order to reach a goal. Besides these properties, mobility, benevolence and mental states can also be considered [46].

According to the social skill property, agents can interact and cooperate among themselves to reach the goals. Multiagent systems (MAS) are based on such coordinated cooperation, or collaboration, to reach goals. Therefore, a MAS can be seen as a set of social organizations of agents, resulting in a society. In such organizations, agents interact by means of negotiation and cooperation, coordinating the activities to reach goals of the society to which they belong. The organizations are dynamic configurations of relationships among agents, resulting in a system with qualities or characteristics that cannot be observed based only on the individuals [22].

The dynamic aspects can be mapped to relationship changes among agents and the addition and removal of agents, with new skills, thus defining an open system. These changes may influence their isolated actions, introducing new functionalities or services. Therefore the agent design must be flexible enough to support such organizational changes.

Many solutions have been introduced in the literature to the design and integration of MAS, from methodologies to computational tools, such as the MadKit [33], AgentTool [38], FIPA-OS [20], JADE [4], Zeus [48], among others. Most of these solutions usually emphasize the structure and capabilities of an isolated agent [17], or when cooperation among agents is taken into account, particularly in heterogeneous environments, the relationships are statically defined. Thus, the agents maintain the same services and the interactions with other agents during all the life cycle of the system.

Considering the need to provide support for the development of MAS based applications, with emphasis on the inherent open and dynamic characteristics of such systems, we introduce in this paper an infrastructure named COMPOR. More specifically, we describe a methodology (COMPOR-M), a component model (COMPOR-CM), a component based framework (COMPOR-F), and an environment (COMPOR-E) for development multiagent systems.

The conceptual basis for the COMPOR methodology is the MATHEMA society of agents [14] and the component based software development $[15,16]$. We introduce guidelines to build multiagent systems, resulting in systems that are flexible enough to dynamically adapt to requirements changes, that are reflected both in the relationships in the society of agents as well as in the services provided by the agents. It must be emphasized that the focus on this paper is on the software engineering aspects related to the design of MAS, the reader may refer to [22] for other aspects and concerns.

The environment provides tools to build multiagent systems based on the COMPOR methodology, covering from the analysis to test phases. More specifically, the designer using the environment can execute different activities related to the development cycle of MAS. Among such activities the specification of the problem can be graphically defined and the skills to solve the problem can be defined. In the context of this paper, an skill is defined as the knowledge and techniques needed to solve a problem for a specific context. Besides, the environment also provides mechanisms to map contexts to organizations and skills to agents, thus resulting in a society of agents. After that, the designer identifies the functional components for the agents, uses the environment to integrate them and may execute interaction tests. Finally, the mechanisms to monitor and execute the system are defined, and then, the relationships among agents in the society are defined. 
Each one of the activities is detailed in the rest of the paper. Critical discussions about related works are presented, specifically in Section 3.5 other methodologies are discussed, in Section 4.1 other works related to frameworks to develop MAS are discussed, and in Section 5 other tools are discussed. The remaining of the paper is organized as follows: in Section 2 the MATHEMA model for domain analysis is discussed; in Section 3 the methodology, the guidelines, the techniques and diagrams are introduced; in Section 4 the component model, named COMPOR-CM, as well as the framework (COMPOR-F) to build multiagent systems is introduced, while in Section 5 development environment (COMPOR-E) is detailed; in Section 6 , in order to illustrate the methodology application, a case study in the context of electronic commerce negotiation is shown and finally in Section 7 the conclusions are presented.

\section{The MATHEMA Agents Conceptual Model}

As said in the introduction the conceptual basis for the COMPOR methodology is the MATHEMA model [14], as shown in Figure 1(a). This model is based on a tri-dimensional view of the problem knowledge domain, that was initially defined for the context of multiagent intelligent tutoring systems (ITS) [14] and has been applied in many different domains, such as algebra [10], logics [14], Petri nets [9], and musical harmony [11].

After the design of different ITS applications [13] based on the MATHEMA conceptual model, we observed that this model could be applied to develop other kind of multiagent systems as well. A knowledge domain is denoted by $D$ and is partitioned in contexts and depths, defining sub-domains, namely $D_{i j}$. The domain may have lateralities as knowledge support.

For instance, in [14] it is introduced an ITS for the classical logic domain, where: $D=$ classical logic, and three context are defined, namely: $C_{1}=$ axiomatic vision; $C_{2}=$ natural deduction vision; and $C_{3}=$ algebraic vision. Possible depth and lateralities are: $D_{11}=$ propositional logic, and $L_{111}=$ set theory. The definition of the society of agents based on this tri-dimensional view is obtained mapping each pair of (context, depth) to agents. The lateralities are also mapped to agents, as shown in Figure 1(b).

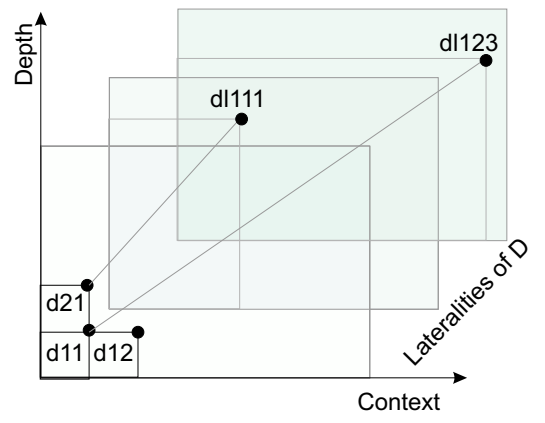

(a)

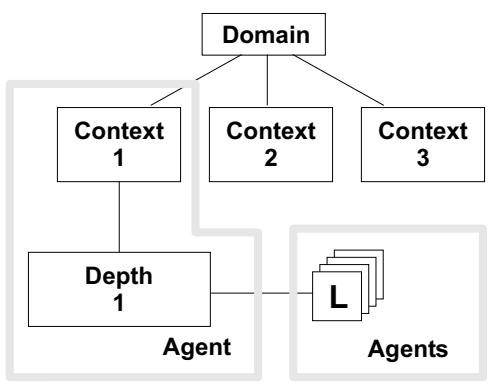

(b)

Figure 1: Tri-dimensional view of the knowledge [14]

To generalize the model for other MAS applications the laterality and depth concepts were redefined in a unique concept named skill. A skill is a set of knowledge and techniques necessary to execute a given task defined for a specific context. Therefore, the skill concept encapsulates a pair (context, depth) or a laterality. This is the analysis basis for the COMPOR methodology introduced in Section 3. 


\section{The Methodology}

The methodology to develop multiagent systems introduced in this paper is defined based on the same four classic phases adopted for other kind of software development, namely, analysis, design, implementation, and tests. The development is iterative and with strong incremental characteristics as a consequence of the poor, imprecise, or incomplete requirements definition - an inherent characteristic of dynamic open systems such as MAS. The applicability of this kind of approach is increasing since the requirements of such systems are becoming more mutable during the design phase. The development cycle for the methodology introduced in this paper is illustrated in Figure 2, and each phase is described in the following sections.

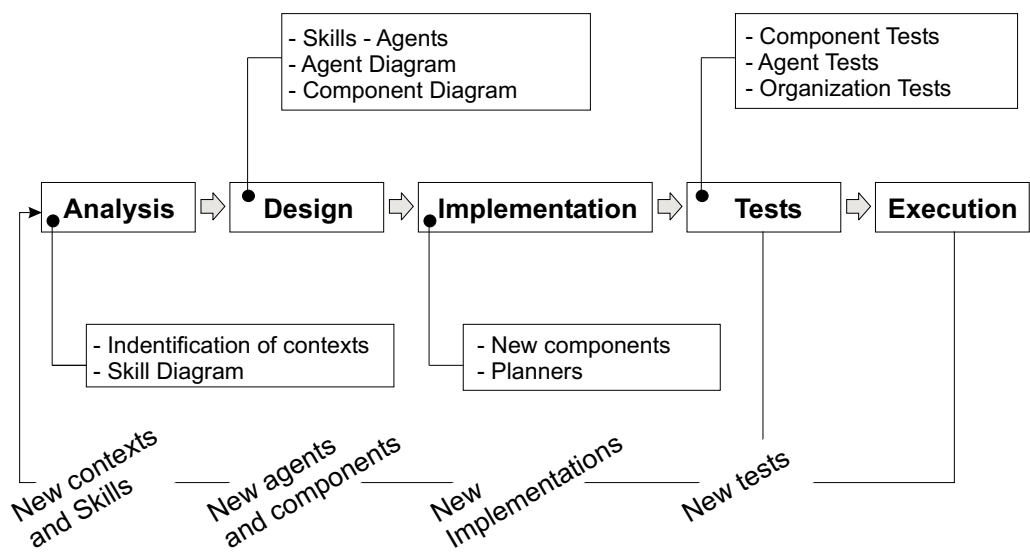

Figure 2: Development cycle for the COMPOR methodology

\subsection{Analysis Phase}

As said before the analysis phase is based on the domain partition defined for the MATHEMA conceptual model. The guidelines were generalized to deal with different kinds of application domains for MAS: (i) given a problem knowledge domain, the contexts are defined; (ii) based on the contexts, the skills needed to each context in order to solve problems are identified, and; (iii) the skills for the contexts are described using the skill diagram as shown in Figure 3. Associations among skills should be defined in this diagram.

\subsection{Design Phase}

When the skills for each context are defined, the architecture of the agents can then be defined. The first step is to map the skills to agent attributes. The second step is to generate the agent diagram as illustrated in Figure 4. For this diagram, the contexts identified in the analysis phase are defined by organizations of agents that are represented by entities with context skills. Besides this, the interaction role of agents should be defined, according to protocol that will rule the interaction among agents.

The grouping of agents in organizations characterizes a set of agents according to a given context, where the interactions will be more frequent [35]. The interaction roles and skills for the agents are defined, as well as the organizations for them, the internal architecture for the agents can then be defined in terms of the functional components. As said before the COMPOR component model, introduced in Section 4, is based 


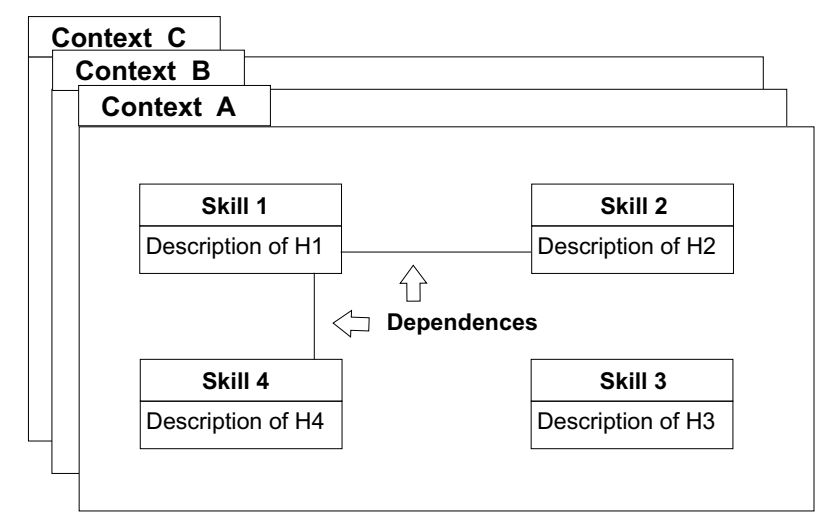

Figure 3: Skill Diagram

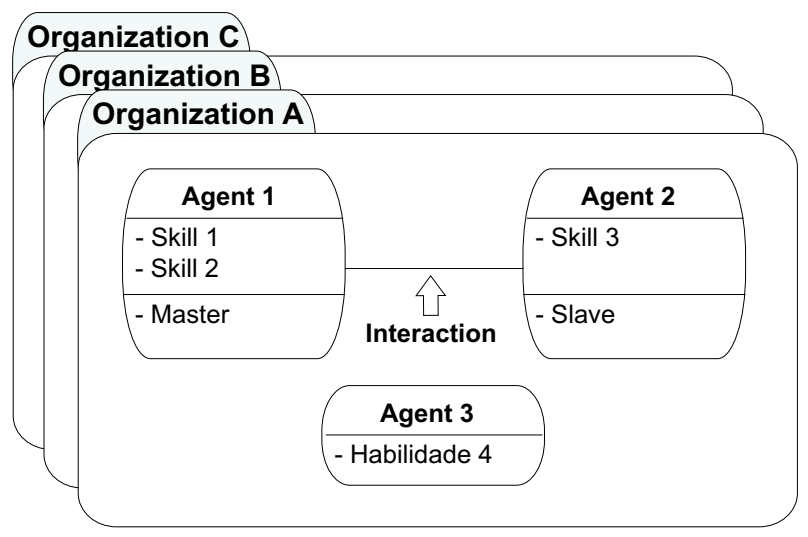

Figure 4: Agent diagram

on the agent model defined in [14]. Thus, an agent is composed by three different abstract systems that represent functionality contexts: (i) intelligent or deliberative system:includes the functionalities for a given application domain and features such as autonomy, learning, knowledge inference, among others; (ii) social system: implements the interaction functionalities, such as protocols and beliefs, and; (iii) distribution system: includes the communication functionalities based on different technologies, such as RMI [41], CORBA [32], and SOAP [6].

In Figure 5 it is illustrated the organization of the society of agents according to the MATHEMA model and adapted to the COMPOR [12].

Based on the introduced architecture the diagram of components is then obtained. Such diagram defines the agents of the society (the set of all organizations) and the components for each agent systems (intelligent, social, and distribution). The names for these components must be written in the spaces reserved for each one of the systems in the diagram, as shown in Figure 6. In Section 4 the guidelines to specify the components are detailed. The design phase is completed as soon as all the agents and its functional components are defined. 


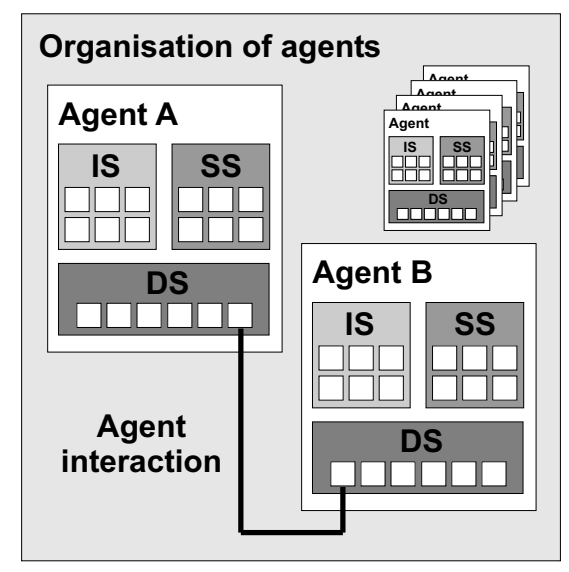

Figure 5: Organization of the society of agents according to the MATHEMA model

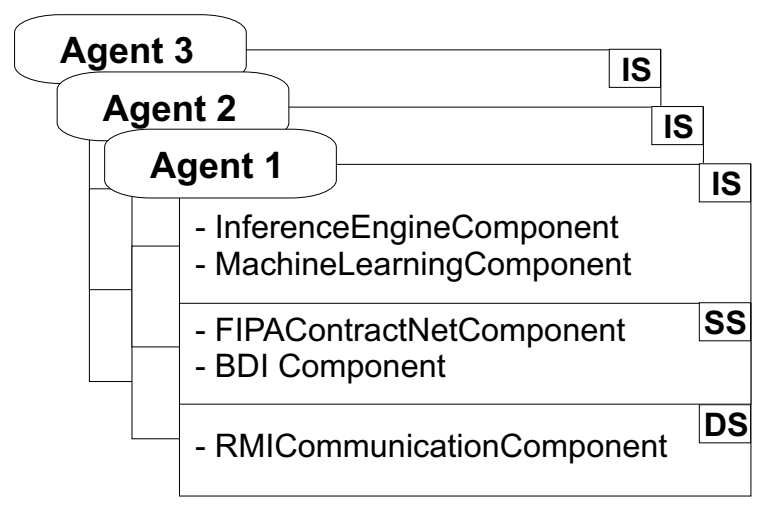

Figure 6: Component Diagram

\subsection{Implementation Phase}

In this phase the components defined in the design phase are implemented. The use of the COMPOR-CM component model, detailed in Section 4 is mandatory for this phase. This is due to the fact that without a model defining the interaction among the components, and without the library of components, all the components would have to be implemented from scratch, as well as the definition for the interactions. Based on the model, the focus on the implementation phase is on building components that are not available in the library and the definition of the interactions. Besides this, an environment to aid during this process is available to the designer, namely, the COMPOR-E environment that is presented in Section 5.

\subsection{Test Phase}

As said before, the functionalities of the agents are provided by components, thus unit and functional tests for each component are independent from the tests related to the agents and the society. The developed components must be tested independently, and preferably using a test automation framework. The test guidelines introduced in this paper are restricted to agents and organizations. In the first case the interactions 
tests are performed among the components of a given agent, and in the second case the interactions among the agents in a given organization of a society is performed. It is important to point out that the effective use of tests depends on the availability of a tool to automatize them. Therefore, the COMPOR-E environment (see Section 5), provides a tool for this purpose.

\subsection{Other Works Related to the Methodology}

Different methodologies have been proposed to the systematic development of MAS. Most of these methodologies are based on object oriented concepts such as classes and inheritance, with some adaptations to agent oriented software engineering, such as Gaia [47], MASE [17], Burmeister [7], Cassiopeia [19], SODA [43], among others. For these approaches the society of agents is defined based on inter-relationships or roles identified during the analysis phase. These relationships among roles are then transformed to interactions among the agents. The notion of roles is similar to the notion of skills defined in this paper, but it is important to point out that the relationships among roles are static. There is no redefinition of the relationships among roles reflecting the interactions among the agents in a society. For multiagent systems the nature of the interactions are inherently dynamic. In some cases, as for instance for the example introduced in Section 6, it may be the case that an exact definition of the requirements cannot be obtained, and therefore, the relationships among roles for the agents. The approach introduced in this paper, based on skills, is more flexible since the relationships can change.

On the other hand, there are knowledge based methodologies instead of software engineering methodologies such as CoMoMAS [29] and MAS-CommonKADS [34], among others. In these cases the main concern is in the representation of the agents as deliberative entities, defining behavioral and learning rules. The complexity level for the agent entity is much higher, due to the fact that it is not considered only an abstraction entity - as the evolution of an object. The methodology introduced in this paper uses the agent as an abstraction, but assumes that the behavior may or not be deliberative. The behavior of each agent is defined based on the components of the architecture, and thus the deliberative characteristics may be implemented by the components. The entity used to construct the software is an agent that can be as complex or simple as needed.

\section{Framework (COMPOR-F) and the Component Model (COMPOR-CM)}

A component model specifies the patterns and conventions for the developers of components based on the given model. That is, it allows the definition of the interaction rules among components and specification conventions that must be followed to connect new components to the model. In order to implement such interactions in a transparent way, according to [21], a framework based on components is then defined. The framework is an implementation of the services to satisfy the component model [2]. In the remaining of this section we present the component based framework (COMPOR-F) and the specification of the component model (COMPOR-CM). We also introduce a discussion related to other component based frameworks and models introduced to develop MAS in the end of the section.

\subsection{The Component Based Framework}

Since the COMPOR-F framework [12] is based on components, its architecture is more flexible and the solutions more reusable [21]. The functionalities of the agents are provided by the components and the 
interactions among them are provided by the framework. Therefore, the framework is flexible enough to be adapted for different multiagent architectures. The designer may define the functionalities of the agents according to the needs and the specific organization technology, based on a component based development. The framework allows the composition and interaction among agents [15, 16].

The conceptual basis for the framework model is the MATHEMA model, shown in Figure 5, introduced in Section 3. The structure of the model is based on the Composite design pattern [27]. Its functionality can be applied to hierarchical structures, which is the case of the MATHEMA model, as shown in Figure 7. Observe that the entities AgentComponent, AgentContainer and ComponentModel are the basis for the component model for the COMPOR framework. The model is implemented based on JavaBeans [40], with different characteristics as discussed and justified as follows.

\section{Component interaction model - COMPOR-CM}

The interaction model defined for JavaBeans is based on the concept of events, where entities generate events, announce them, and other entities are informed that events occurred. To promote such kind of interaction it is necessary an explicit reference for the involved entities. In the case of the COMPOR-CM model, there are two types of component interaction: based on events and based on services.

In the case of the interaction based on events, the focus is on the announcement of state changes for a component to other involved components, it is based on the observer design pattern [27]. Each component specifies which events have to be announced and those that it is interested on based on a declarative approach. That is, each component has an XML configuration file (descriptor) that defines the events that this component is interested on. Then, the framework implements mechanisms to announce an event for the interested components when this event occurs.

The interaction based on services allows service invocations without explicit references to the service client and provider components. For each component, the provided services are specified in a descriptor. In this way, when another component wants to execute the service, it simply requests the service to the framework, without references to the service provider component.

\section{Asynchronous interaction among components}

Besides the usual synchronous communication, COMPOR-CM provides the necessary infrastructure for asynchronous communication among components. Thus, the services can be requested in a synchronous or asynchronous way, according to the application requirements. It is important to point out that in the case of JavaBeans, this type of interaction must be implemented by the component developer.

The process to define properties initialization, that is, the definition of the properties that must be instantiated so that the component can function correctly, was modified to allow the component developer to define such properties declaratively, using an XML descriptor. One weakness of such approach is that the representation of complex initialization properties may be difficult, whereas in the case of JavaBeans such situation can be better managed using the concept of editors of properties. Nevertheless, the approach adopted promotes an increase in the simplicity, the flexibility and the practical use of the model. 


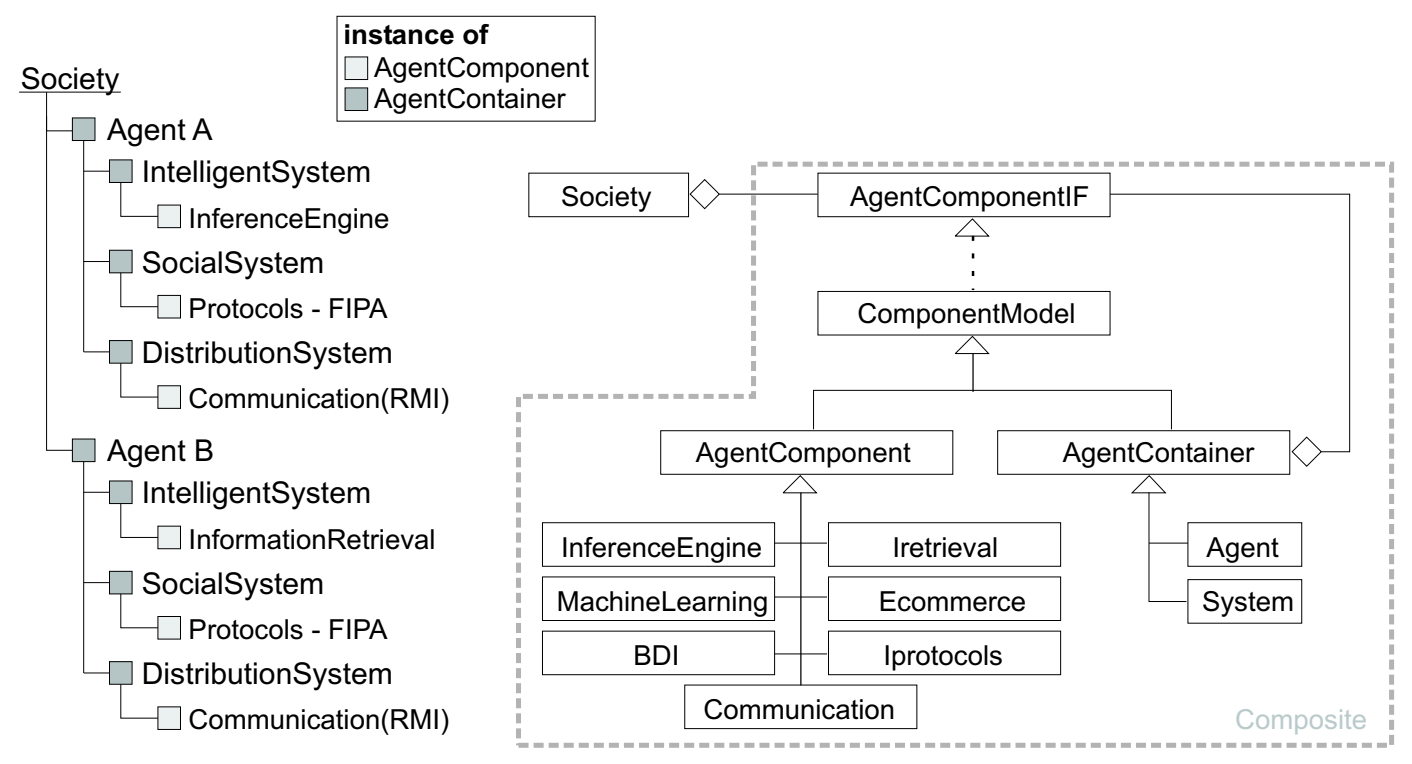

Figure 7: Composite pattern for the MATHEMA model.

\section{Class Diagram}

In Figure 8 the class diagram for the COMPOR-F framework, together with the main methods and attributes, is shown. It is based on the model presented in Figure 5. There are other classes for the whole implementation but for simplicity they are omitted in the diagram shown. Nevertheless, such choice does not compromise the understanding. Besides, emphasis is given on details related to the composite pattern, that is the basis for the design of the framework.

The methods declared in the AgentComponentIF interface have different implementations for AgentComponent and AgentContainer, and their functionalities are describe as follows. Observe that exceptions are not shown.

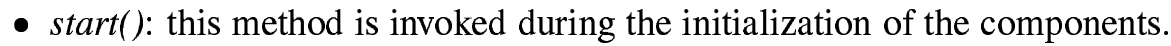

- AgentContainer: initializes all the components that belong to it invoking the method start().

- AgentComponent: invoke a template method [27] that is implemented by the component developer, which initializes the necessary properties allowing its operation.

- stop(): the behavior of this method is similar to the behavior of the method start(). The difference, is that it is invoked when a component has to be stopped.

- $\operatorname{doIt}()$ : this method is invoked when an agent component intends to invoke some service provided for another component defined for the same agent.

- AgentContainer: if the requested service is implemented by some AgentComponentIF that belongs to it, then the execution is forwarded through the doIt invocation for the AgentComponen$t I F$. Otherwise, the service execution is forwarded to the AgentContainer to which this AgentContainer belongs to. 


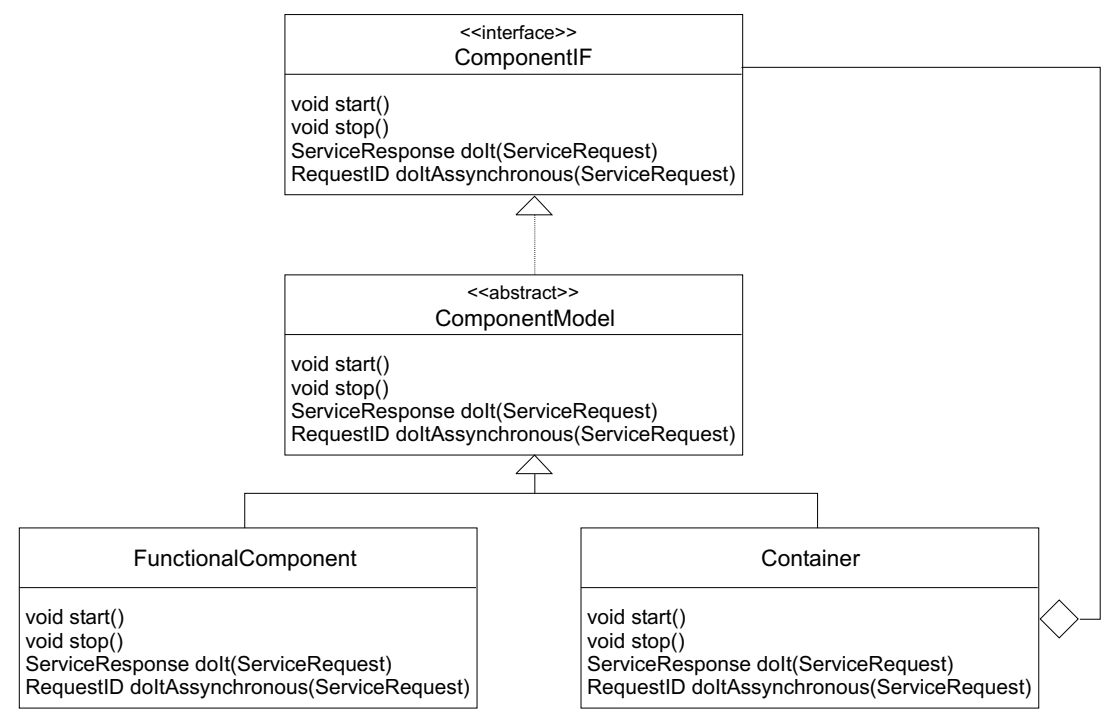

Figure 8: Class diagram for the framework

- AgentComponent: if the requested service is implemented by the invoked component, then it executes the it. Otherwise it forwards the service execution to its AgentContainer.

- doItAssynchronous(): this method is an asynchronous version of the doIt method.

- AgentContainer: this method is not implemented by containers.

- AgentComponent: creates a new thread to execute the method doIt, announcing the return of the request to the service invoker.

\section{Library of Components}

Since components do implement the functionalities of an agent, it is necessary to make available a library or repository of components that can provide the common functionalities of multiagent systems, such as interaction protocols, communication protocols, as well as other cognitive and deliberative functionalities. The specification of COMPOR components is detailed latter in this section. The COMPOR component library makes available RMI and SOAP communication components, JDBC database access, components to model the behavior of learners for intelligent tutoring systems applications, components to access the JESS API [26], among others. Components related to interaction protocols and languages, such as FIPA-ACL [25] and KQML [24]) are being added to the library.

\section{Interaction Scenario}

In order to illustrate the behavior of the interaction model implemented by the framework, and also easy the understanding, components with simple functionalities were developed. Since the framework does not provide the communication infrastructure, then, such structure is provided by components that belong to the components library, and the components developed for this example are the following ones: 
- Sum component: the service provided by this component is the addition of two real numbers.

- Description of the service: "sum"

- Parameters: Double aNumber, Double otherNumber

- Multiplication component: the service provided by this component is the multiplication of real numbers through the Sum service that in this example, is implemented by the Sum component.

- Description of the service: "mul"

- Parameters: Double aNumber, Double otherNumber

Besides these two components, the communication component, that is available in the component library, was used to illustrate the interaction between two agents in the society.

- Communication component: provides communication between two components using the communication network.

- Description of the service: "sendService"

- Parameters: ServiceRequest serviceRequest, where ServiceRequest is an object that encapsulates the service name and its parameters.

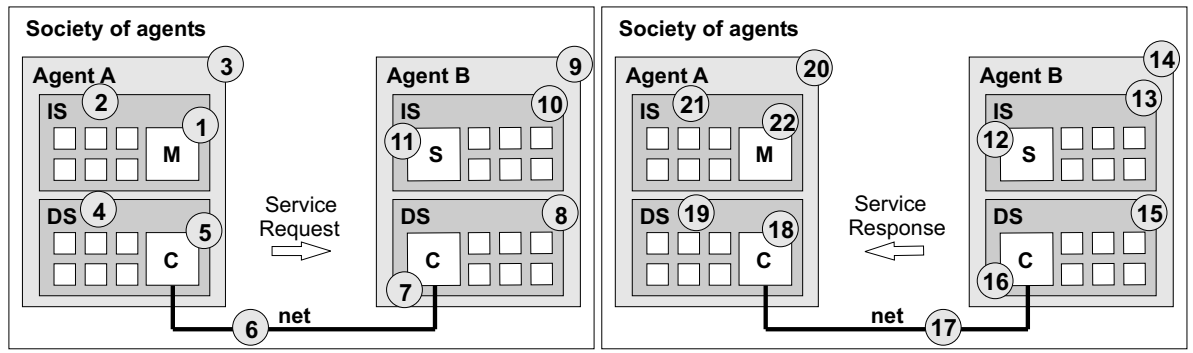

Figure 9: Interaction scenario

The interaction scenario for this example is illustrated in Figure 9, and its behavior is described as follows. There are 22 steps, and from steps 1 to 11 (detailed below) a service request is detailed. The multiplication component $(M)$ for Agent $A$ needs to request the service "sum" that is provided by Agent B. Observe that these two agents are physically distributed. To do so, it must request to the communication component to send a service request to the Agent $B$. This request is made through the invocation of the "sendService" service. Such request contains the service name (sum) and its parameters, to Agent B.

1. Since the multiplication component does not implement the service "sendService", it forwards the execution of doIt to its father-Container, who is the Intelligent System(IS) of the agent.

2. The request arrives at the $I S$ of Agent $A$, which verifies if some of its children-components implement the service. As there is no child-component in the $I S$ that implements the service, then the execution of doIt one more time is forwarded to its father-container, in this case, the Agent A. 
3. Agent $A$, then verifies that the Distribution System $(D S)$ implements the service, and delegates again the execution of doIt.

4. $D S$ is a container and therefore it does not implement any service, however it contains the component which implements this service. Thus, the execution of doIt is forwarded to the CommunicationManager $(C)$ component that implements the "sendService" service.

5. CommunicationManager of Agent A sends a message to the CommunicationManager of Agent B. This message contains the service to be remotely executed (in this example, "sum"), as well as the necessary parameters for its execution.

6. The interaction between the components through the network represents the communication between the agents of the society.

7. CommunicationManager of Agent $B$ receives the message, and extracts the service and parameters to be requested, and then invokes doIt.

8. The execution of doIt is forwarded to Distribution System of Agent B.

9. $D S$ forwards to its father-container(Agent $\mathrm{B}$ ).

10. The container Agent $B$ forwards the request to the $I S$.

11. The IS then forwards the request to the Sum Component $(S)$, that in turn executes the addition and returns the result.

Steps 12 through 22, shown in Figure 9, are not in this paper due to space restriction. These steps represent the return of a service invocation as the result of a request. These steps are related to the returning path from the provider $S$ and the client $M$ of the service, including the communication component. Observe that for all the steps, there is no explicit reference to any component.

\subsection{Component Specification}

In order to easy the development of component based systems it is necessary to clearly specify the functionalities made available for each component as well as use information (contract), allowing the developers to perform black box reuse. It is necessary to provide some specification of the services provided by a component. In what follows we detail the specification model for the COMPOR-CM.

1. Name: the name of the component

2. Description: a brief description of the services provided by the component.

3. Type Dictionary: definition of abstract data types used in the next specification steps.

4. Initialization attributes: initialization attributes of the component.

5. Provided services: a list of the services provided by the component.

6. Required services: a list of the services required for the component. 
7. Announced events: a list of the events announced by the component.

8. Interesting events: a list of the events that are interesting for the component.

The components in the library of COMPOR components, see Section 4.1, follows this specification.

\subsection{Other Works Related to MAS Frameworks}

In order to support the development of MAS, some object-oriented frameworks have been introduced. Although most of them provide the necessary infrastructure for the interaction among agents in a society, there is a lack of flexibility to adopt different technologies, languages or protocols for the interaction among agents. The DECAF framework ${ }^{1}$ [31], for example, provides an implementation of all the agent interaction requirements, but there are no mechanisms to integrate new protocols. In the case of the JADE framework ${ }^{2}$ [4], the protocols are based on FIPA ${ }^{3}$ [42], however the communication among agents is not very flexible. The RMIT framework [37] is composed of eight subframeworks, one for each agent layer, introducing modularity. On the other hand it lacks flexibility, since the messages are only sent between adjacent modules. Also, since the design if rigid it compromises its scalability and maintenance.

Based on this related works, we can observe that the functionalities of the agents, related for example to the cognitive reasoning mechanism, interaction protocols, and communication, are tightly coupled to the framework. The same characteristics were observed in other proposed solutions $([49,5,1,8,39,30])$. Therefore, for these frameworks the design of the agents become dependent of the specific functionalities provided by them.

\section{Development Environment (COMPOR-E)}

A graphical environment has been developed to support the construction, configuration, execution, and monitoring of the agent society. The architecture of the environment was defined to allow the insertion of new tools based on a Java interface and the configuration of a XML descriptor defining the tools available in the environment. Currently the environment have four tools as detailed in what follows.

- Agent building tool: used to define the agent society. In Figure 10.a it is presented the agent tree, where it is possible to add agents, as well as to specify the components that will compose the agents. That is made dragging the components from the palette and dropping these components in the agent tree. Also, the tools has a inspector for tree nodes, allowing the visualization of the defined properties. In Figure 10.b the state of the inspector for the tree in Figure 10.a is shown.

- Component deployment tool: deploys user developed components. In Figure 10 the component palette of the system is presented. The user developed components are added to this palette after deployment.

- Modelling tool: used to aid the developer in the different phases of the methodology introduced in Section 3, and makes available diagram building modules and automatic MAS project generation based on the diagrams. Provides pre-defined architectural templates for both interaction protocols using patterns as well as mental states [44]. Besides this, it provides the execution of automatic tests.

\footnotetext{
${ }^{1}$ Distributed, Environment-Centered Agent Framework

${ }^{2}$ Java Agent Development Framework

${ }^{3}$ Foundation of Intelligent Physical Agents
} 
- Execution and monitoring tool: used to control and to manage the execution of the local and distributed agents.

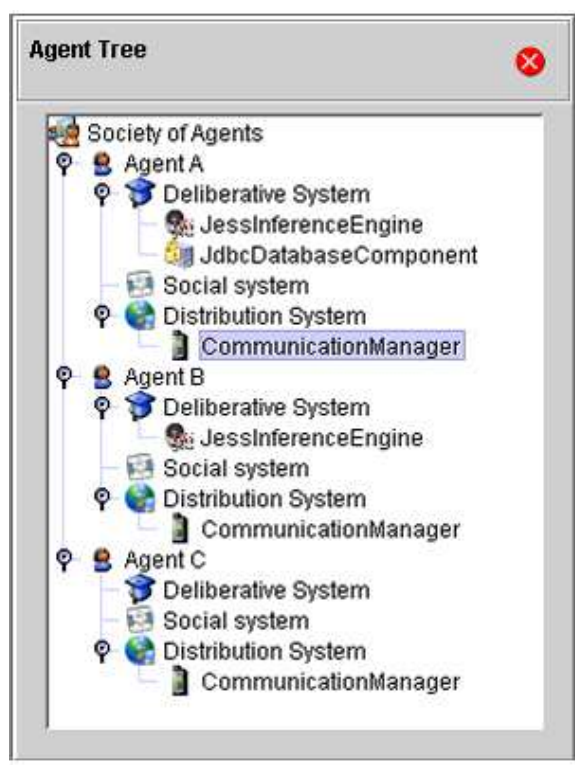

(a)

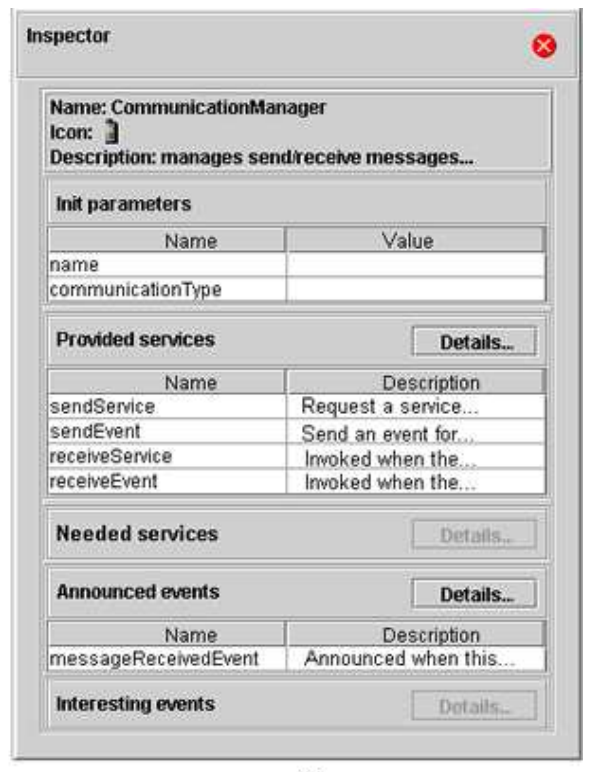

(b)

Figure 10: Tools to construct agents

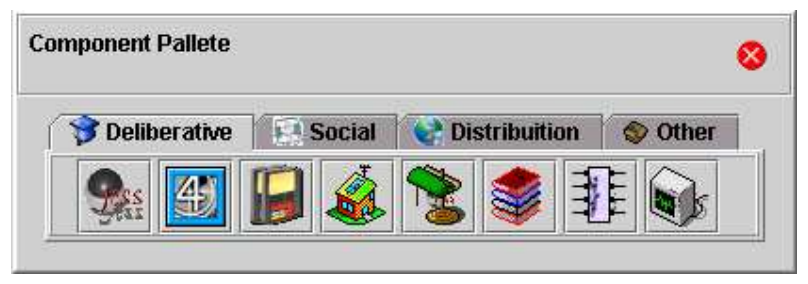

Figure 11: Tool to deploy components

Using the environment a designer can: specify the problem and its knowledge domain based on graphical models; define the skills needed for different contexts of domain to solve problems; map the contexts to organizations and agent skills, thus defining a society of agents; identify the functional components that will compose the agents; test the interaction among agents; and, monitor the execution.

\section{Other Related Development Tools}

Some tools have been proposed to agent oriented software development based on the methodologies mentioned before. The AgentTool [38], for instance, provides a development environment based on the MASE methodology [17]. JATLite [36] and FIPA-OS [20] provide the interaction infrastructure for agents and Zeus [48] and the MADKit [33] are tools to construct agents based on the definition of the behavior of 
each agent for a given organization. The COMPOR-E environment provides analysis tools that are similar to those of the AgentTool, but it is based on the methodology introduced in this paper instead. And as we argued before incorporate better features to promote the development of multiagent systems. With respect to JATLite, COMPOR-E provides the communication infra-structure based on its components, and therefore is more flexible, because the developer may define it. Compared to Zeus, COMPOR-E is based on the organization of the agents, while in Zeus it is assumed that an agent already has all of its tasks pre-defined and its interface guarantees this, and therefore its applicability is restricted to systems where the tasks to be executed are well known a priori. In the case of COMPOR-E, an agent is not merely a task executer, instead, it is a container of components, that if needed, may implement a tasks execution behavior.

\section{Case Study}

In order to validate the methodology and to identify possible necessary improvement for the guidelines as well as the environment interface, applications are being developed. In this paper we discuss the development an electronic commerce negotiation tool, with emphasis on the modelling of the organization where the relationships among the entities cannot be completely defined during the requirements phase [23]. The focus is on the negotiation model, and we assume that the behavior of the enterprise is more complex than a simple opponent concept as described in [18]. For the business model the initial relationships are illustrated in Figure 12. Observe that they cannot be completely predicted and fixed during the life cycle of the enterprise, as well as for the computational negotiation system life cycle. Besides, the system must be dynamic enough to adapt to relationships changes. It is important to point out that new relationships may be necessary during the life cycle of the enterprise. For instance, it might be necessary to monitor the behavior of the currency exchange market if the enterprise starts selling foreign products.

\subsection{Analysis}

Based on Figure 12, one can notice that many different contexts are related to the enterprise domain: (i) negotiation relationship between the Consumer and the Enterprise can be defined in a negotiation context, where the necessary knowledge is related to the definition of negotiation proposals and counter-proposals; (ii) the relationships $R 1$ to $R 5$ can be respectively mapped to the Investors context, monitoring the investments of the enterprise, the Government context, monitoring the taxes and fees applied to products made or negotiated by the enterprise, Competitors context, monitoring the prices and advantages given by the competitors, Suppliers context, monitoring the prices and payment conditions offered by the suppliers; and

finally the Consumer, that monitors the history of the clients. Based on the identification of these contexts the skill diagram is obtained, as shown in Figure 13.

\subsection{Design}

After defining the skills needed for each relationship, the organizations to support the skills can be defined. In Figure 14, it is shown the agent diagram for the case study. When the agents, their goals, and skills are defined, as well how they are organized, then the architecture based on components of each agent can be defined. In Figure 15 it is shown the component diagram for the negotiation MAS. For simplicity the agents have a unique component architecture, and all of them can access the database to recover the information necessary for each monitoring activity. 


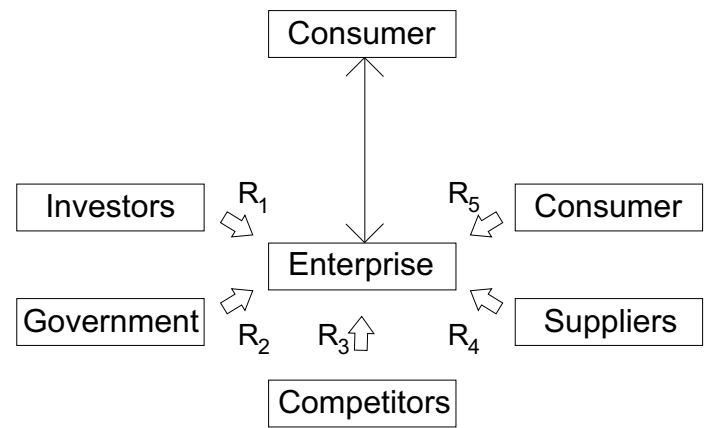

Figure 12: Modelling of business relations in an enterprise

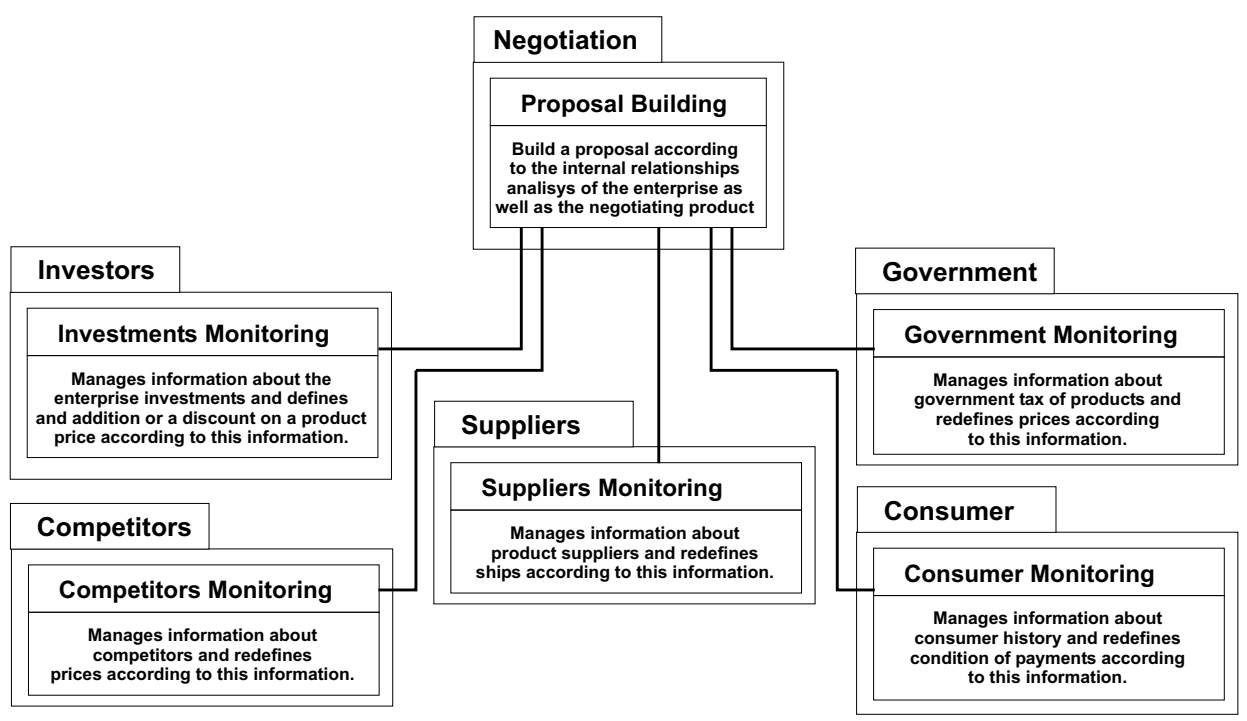

Figure 13: Skill diagram for negotiation

In the implementation and test phases the COMPOR-E development tools were used. The tools provide the required components to build the society of agents, and as said before, this is a "drag and drop" process. The agents are built and the services started. The access to the services available in the multiagent society may be done by the Facade design pattern [27], implemented by an Interface Agent, which encapsulates all the service calls of the society. The organization tests and the agents in the interface of the tool uses as the basis the JUnit framework [3].

\section{Conclusions}

In this paper we introduced the COMPOR infrastructure - a set of guidelines, techniques and tools to develop multiagent software systems. In the definition of the methodology the inherent dynamic and open nature of such systems was taken into account, considering an incremental development process, without the need to define a priori all the relationships among agents in a society. To do so, we used a model for compo- 


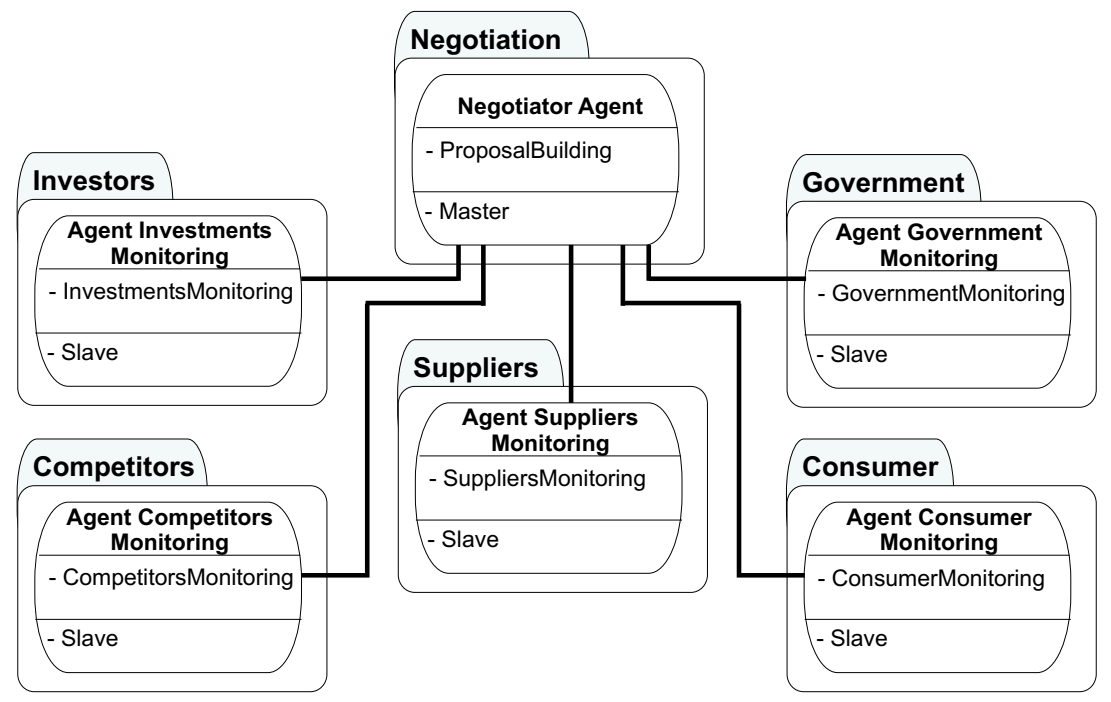

Figure 14: Agent diagram for the negotiation

\begin{tabular}{|c|c|c|c|}
\hline Agent-Monitor & IS & Agent-Negotiator & IS \\
\hline $\begin{array}{l}\text { - MonitoringComponent } \\
\text { - JDBCAccessPoint }\end{array}$ & & $\begin{array}{l}\text { - NegotiationComponent } \\
\text { - InferenceEngineComponent }\end{array}$ & \\
\hline - SocialKnowledgeComponent & & - SocialKnowledgeComponent & $\mathbf{S S}$ \\
\hline - SoapCommunicationComponent & DS & - SoapCommunicationComponent & DS \\
\hline
\end{tabular}

Figure 15: Component Diagram for the negotiation

nents and a component based framework allows changes of the relationships and the skills for the agents, without interfering in the execution of the system. Besides this, we introduced an environment to aid in the development and execution of systems built based on the COMPOR methodology.

From the implementation point of view we are enriching the development environment, finishing a test tool to allow the execution of organization and agents tests in a systematic and assisted way. As future research directions, we intend to develop other applications, besides the already developed one, as the case study described in this paper. Such experiments will allow the definition of methodological specifications improvements as well as in the models for the components and for the framework.

\section{References}

[1] Henri Avancini and Analía Amandi. The Open Agent Architecture: a framework for building distributed software systems. SADIO Electronic Journal of Informatics and Operations Research, 3(1):1-12, 2000.

[2] F. Bachman. Technical concepts of component-based software engineering. Technical report, Carnegie Mellon - Software Engeneering Institute, May 2000.

[3] Kent Beck and Erich Gamma. Junit test infected: Programmers love writing tests - java report. Technical report, 1998. 
[4] F. Bellifemine, G. Rimassa, and A. Poggi. Jade - a fipa-compliant agent framework. In Proceedings of the 4th International Conference and Exhibition on the Practical Application of Intelligent Agents and multiagents, UK, pages 97-108, 1999.

[5] Calebe Paula Bianchini, Diogo Sobral Fontes, and Antonio Francisco Prado. A distributed software agents platform framework. In 1st International Workshop on Software Engineering for Large-Scale multiagent Systems - SELMAS'2002 - Orlando/Florida - USA, 2000.

[6] Don Box, David Ehnebuske, Gopal Kakivaya, Andrew Layman, Noah Mendelsohn, Henrik Frystyk Nielsen, Satish Thatte, and Dave Winer. Simple object access protocol - w3c note 08. Technical report, W3C, May 2000.

[7] B. Burmeister. Models and methodology for agent-oriented analysis and design. In Working Notes of the KI'96 Workshop on Agent-Oriented Programming and Distributed Systems, DFKI Document D-96-06 - Saarbrücken Alemanha. 1996.

[8] Deepika Chauhan. JAFMAS: A Java-based Agent Framework for Multiagent Systems Development and Implementation. PhD thesis, ECECS Department Thesis, University of Cincinnati, Cincinnati, OH, 1997.

[9] E. B. Costa, G. M. Góis, J. C. A. Figueiredo, and A. Perkusich. Towards a multiagent interactive learning environment oriented to the petri net domain. In In Proc.of IEEE Int. Conf. on Systems Man and Cybernetics, pages 250-261, San Diego, USA, October 1998. Springer-Verlag.

[10] E. B. Costa, M. A. Lopes, and E. Ferneda. Mathema: A learning environment based on a multiagent architecture. In J. Wainer and A. Carvalho, editors, Proceedings of the 12th Brazilian Symposium on Artificial Intelligence, volume 991 of Lecture Notes in Artificial Intelligence, pages 141-150, Campinas, Brazil, 1995. Springer-Verlag.

[11] E.B. Costa, H. O. Almeida, E. F. A. Lima, R. R. G. Nunes Filho, K. S. Silva, and F. M. Assunção. A cooperative intelligent tutoring system: The case of musical harmony domain. In C. Coello, A. Albornoz, L. Sucar, and O Battistuti, editors, Proceedings of 2 nd Mexican International Conference on Artificial Intelligence - MICAI'02, volume 2313 of Lecture Notes in Artificial Intelligence, pages 367-376, Mérida - Yucatán - México, April 2002. Springer Verlag.

[12] Evandro Barros Costa, Hyggo Oliveira Almeida, Rodrigo de Barros Paes, and Angelo Perkusich. Compor: a component-based framework for building multiagent systems. In Proceedings of 2nd International Workshop on Software Engineering for Large-Scale Multiagent Systems - on ICSE 2003 - Portland - USA, pages 84-89, May 2003.

[13] Evandro Barros Costa, Hyggo Oliveira Almeida, Klebson Santos Silva, and Angelo Perkusich. A conceptual framework for building intelligent tutoring systems in a distance learning environment based on a multiagent approach. In Proceedings of Workshop Architectures and Methodologies for Building Agent-based learning Environments - ITS 2002 - San Sebastian - Espanha, pages 48-57, June 2002.

[14] Evandro Barros Costa, Angelo Perkusich, and Edilson Ferneda. From a tridimensional view of domain knowledge to multiagent tutoring system. In Proceedings of 14th Brazilian Symposium on Artificial Intelligence - Lecture notes on artificial intelligence, LNAI 1515, volume 991, pages 61-72, Porto Alegre - RS - Brazil, November 1998. Springer Verlag.

[15] Ivica Crnkovic. Component-based software engineering - new challenges in software development. Software Focus, 2(4):127-133, 2001.

[16] Ivica Crnkovic, Brahim Hnich, Torsten Jonsson, and Zeynep Kiziltan. Specification, implementation, and deployment of components. Communications of the ACM, 45(10):35-40, 2002.

[17] S. Deloach, M. Wood, and C. H. Sparkman. Multiagent systemas engineering. In International Journal of Software Engineering and Knowledge Engneering, volume 11, pages 231-258. World Scientific Publishing Company, 2001. 
[18] G. DePaula, G. Ramos, and G. Ramalho. Bilateral negotiation model for agent-mediated electronic commerce. In In Proceedings of Third Workshop on Agent-Mediated Electronic Commerce, pages 1-14, 2001.

[19] A. Drogoul and J. D. Zucker. Methodological issues for designing multiagent systems with machine learning techniques: capitalizing experiences from the robocup challenge. Technical report, LIPS Research Reports, October 1998.

[20] Emorphia. Fipa-os. http://fipa-os.sourceforge.net, 2003.

[21] Mohamed Fayad, Ralph Johnson, and Douglas Schmidt. Building application frameworks. Wiley, 2000.

[22] Jacques Ferber. Multi-Agent System: An Introduction to Distributed Artificial Intelligence. Addison-Wesley, 1999.

[23] Ricardo Rubens Gomes Nunes Filho, Evandro Barros Costa, and Hyggo Oliveira Almeida. An Architecture and A Decision-Making Model to Web-Based Electronic Commerce. In Proceedings of of I3E - 3rd IFIP conference on e-Commerce, e-Business, and e-Government - São Paulo - Brasil, pages 117-128, 2003.

[24] T. Finin, R. Fritzson, D. McKay, and R. McEntire. KQML as an Agent Communication Language. In N. Adam, B. Bhargava, and Y. Yesha, editors, Proceedings of the 3rd International Conference on Information and Knowledge Management (CIKM'94), pages 456-463, Gaithersburg, MD, USA, 1994. ACM Press.

[25] Foundation for Intelligent Physical Agents. Fipa acl message structure specification. http://www.fipa.org/, 2003.

[26] Ernest Friedman-Hill. Jess - the rule engine for the java platform. http://herzberg.ca.sandia.gov/jess/, 2002.

[27] Erich Gamma, Richard Helm, Ralph Johnson, and John Vlissides. Design Patterns: elements of reusable objectoriented software. Addison-Wesley, 1995.

[28] Alessandro Garcia and Carlos Lucena. Software engineering for large-scale multiagent systems, selmas 2002, post-workshop report. Technical report, ACM Software Engineering Notes, August 2002.

[29] N. Glasser. The comomas approach: From conceptual models to executable code. In Proc. of 8th European Workshop on Modeling Autonomous Agents in a multiagent World (MAAMAW-97), Ronneby - Suécia, 1997.

[30] Steven Goldsmith, Shannon Spires, and Laurence Phillips. Object frameworks for agent system development. In Software Tools for Developing Agents - AAAI Workshop, pages 107-112, July 1998.

[31] J. Graham and K. Decker. Towards a distributed, environment-centered agent framework. In In Proc. of International Workshop on Agent Theories, Architectures, and Languages (ATAL99), pages 290-304, 1999.

[32] Object Management Group. Corba faq and resources. http://www.omg.org, 2003.

[33] Olivier Gutknecht, Jacques Ferber, and Fabien Michel. multiagent development kit. http://www.madkit.org/, 2003.

[34] C. A. Iglesias, M. Garijo, and J. C. Gonzales. Analysis and design of multiagent systems using mas-commonkads. In M.P. Singh, A.S. Rao, and M.J. Wooldridge, editors, Intelligent Agents IV (ATAL'97), volume 1365 of Lecture Notes in Artificial Inteligence, pages 314-327, Berlim - Alemanha, 1998. Springer Verlag.

[35] Nicholas Jennings and Michael Wooldridge. Agent-oriented software engineering. In Handbook of Agent Technology. AAAI/MIT Press, 2000.

[36] Heecheol Jeon, Charles Petrie, and Mark R. Cutkosky. Jatlite: A java agent infrastructure with message routing. IEEE Internet Computing, 4(2):87-96, July 2000.

[37] E. A. Kendall, C. V. Pathak P.V. M. Krishna, and C.B.Suresh. A java application framework for agent based systems. ACM Computing Surveys Symposium on Application Frameworks, 2000. 
[38] Multiagent \& Cooperative Robotics Lab. Agent tool project. http://www.cis.ksu.edu/ sdeloach/ai/agentool.htm, 2003.

[39] D. Martin, A. Cheyer, and D. Moran. The Open Agent Architecture: a framework for building distributed software systems. Applied Artificial Intelligence, 13(1/2):91-128, 1999.

[40] Sun Microsystems. Java beans specification. http://java.sun.com/products/javabeans/docs/spec.html, 1997.

[41] Sun Microsystems. Remote method invocation. http://java.sun.com, 2003.

[42] P.D. O'Brien and R.C. Nicol. Fipa - towards a standard for software agents. BT Technology Journal, 16(3):51-59, July 1998.

[43] A. Omicini. Soda: Societies and infrastructures in the analysis and design of agent- based systems. Limerick, Ireland, 2000.

[44] A. S. Rao and M. P. Georgeff. BDI-agents: from theory to practice. In Proceedings of the First Intl. Conference on Multiagent Systems, pages 312-319, San Francisco, June 1995.

[45] Stuart Russel and Peter Norvig. Artificial Intelligence - A modern approach. Prentice-Hall, 1995.

[46] Michael Wooldridge and Nicholas Jennings. Intelligent agents: Theory and practice. In Knowledge Engineering Review, volume 10, pages 115-152. 1995.

[47] Michael Wooldridge, Nicholas R. Jennings, and David Kinny. The gaia methodology for agent-oriented analysis and design. Autonomous Agents and multiagent Systems, 3(3):285-312, 2000.

[48] Zeus. Zeus agent system. http://more.btexact.com/projects/agents/zeus/index.htm, 2003.

[49] Alejandro Zunino and Analía Amandi. Brainstorm/J: a Java framework for intelligent agents. In Proc. of the $2^{\text {nd }}$ Argentinian Symposium on Artificial Intelligence (ASAI 2000 - $29^{\text {th }}$ JAIIO), pages 43-58, Tandil, Buenos Aires, Argentina, 2000. 